\title{
Cloning, Expression and Characterization of a Peptibody To Deplete Myeloid Derived Suppressor Cells in a Murine Mammary Carcinoma Model
}

khadijeh ramezani-ali akbari

Shahid Beheshti University of Medical Sciences School of Medicine

Vahid Khaki-Bakhtiarvand

Shahid Beheshti University of Medical Sciences School of Medicine

Jafar Mahmoudian

Avicenna Research Institute

Hossein Asgarian-Omran

Mazandaran University of Medical Sciences

Fazel Shokri

Tehran University of Medical Sciences

Mohammad Hojjat-Farsangi

Karolinska University Hospital: Karolinska Universitetssjukhuset

Mahmood Jeddi-Tehrani

Avicenna Research Institute

Mahdi Shabani ( $\square$ msshabani@yahoo.com)

Shahid Beheshti University of Medical Sciences School of Medicine

\section{Research Article}

Keywords: immunotherapy, MDSC, peptibody, tumor model, tumor microenvironment, 4T1

Posted Date: December 28th, 2021

DOI: https://doi.org/10.21203/rs.3.rs-1033242/v1

License: (9) (i) This work is licensed under a Creative Commons Attribution 4.0 International License. Read Full License 


\section{Abstract}

Myeloid derived suppressor cells (MDSCs) are an immature heterogeneous population of myeloid lineage that attenuate the anti-tumor immune responses. Depletion of MDSCs has been shown to improve efficacy of cancer immunotherapeutic approaches. Here, we produced and characterized a recombinant peptibody capable of recognizing and depleting murine MDSCs. Using SOE-PCR, the coding sequence of the MDSC binding peptide and linker were synthesized and then ligated into a home-made expression plasmid containing mouse IgG2a Fc. The peptibody construct was transfected into $\mathrm{CHO}-\mathrm{K} 1$ cells by lipofectamine 3000 reagent and the resulting fusion protein was purified with protein $\mathrm{G}$ column and subsequently characterized by ELISA, SDS-PAGE and immunoblotting. The binding profile of the peptibody to splenic MDSCs and its MDSC depletion ability were then tested by flow cytometry. The purified peptibody appeared as a $70 \mathrm{kDa}$ band in Western blot. It could bind to $98.8 \%$ of splenic CD $11 \mathrm{~b}^{+} / \mathrm{Gr}-1^{+}$MDSCs. In addition, the intratumoral MDSCs were significantly depleted after peptibody treatment compared to their PBS-treated negative control counterparts $(P<0.05)$. In this study, a peptibody capable of depleting intratumoral MDSCs, was produced. Our results imply that it could be considered as a potential drug effective for immunotherapy of cancers.

\section{Introduction}

Myeloid derived suppressor cells (MDSCs) are known as an immunosuppressive population of myeloid lineage that are enriched in cancer and chronic infections [1, 2]. MDSCs are primarily generated from myeloid precursors of bone marrow in response to tumor-derived factors such as VEGF, TGF- $\beta$, IL-6, IL-10, CSF-1 and GM-CSF, and are subsequently recruited to blood, spleen and tumor bed [3]. MDSCs are functionally distinguished from the normal myeloid cells based on their ability to inhibit the immune responses mediated by T cells, B cells and natural killer (NK) cells [4-6]. These immune inhibitory effects of MDSCs mostly contribute to expression of the regulatory mediators including arginase1, S100A8/A9, NO, reactive oxygen species, immunosuppressive cytokines and surface immune checkpoints [7]. Moreover, MDSCs facilitate metastasis of primary tumors to distant organs through formation of premetastatic niches [8]. MDSCs are involved in matrix remodeling by induction of MMP8 and MMP9, angiogenesis process and support the metastatic potency of circulating cancer cells $[9,10]$. In a recent study it was reported that epigenetic therapy could disrupt the pulmonary premetastatic niches in murine tumor models by down-regulation of CXCR2 and CCR2 chemokine receptors that mediate the MDSC migration to peripheral organs [11]. Overall, MDSCs take part in the immune escape, invasion and progression of tumors to other organs $[12,13]$.

MDSC-targeting agents are involved in diverse mechanisms to overcome immunosuppression mediated by MDSCs by interfering in MDSC differentiation, hampering their recruitment to tumor site, depleting MDSCs and by reprogramming MDSCs in tumor microenvironment [14]. In this context, early studies demonstrated that all-trans retinoic acid (ATRA) induce differentiation of MDSCs into mature macrophages and DCs and augment the efficacy of anti-VEGFR2 therapy in a breast cancer animal model $[15,16]$. Besides, cytotoxic chemotherapeutic agents including 5-fluorouracil, carboplatin, paclitaxel or gemcitabine have been shown to diminish MDSCs in blood circulation and improve anti-tumor immune responses [17-20]. However, these 
drugs reduce other rapidly proliferating cell populations such as T cells and highlight the need for development of more specific agents without off-targeting effects. Francis Mussai et al. discovered depleting effects of an immunotoxin, anti-CD33 monoclonal antibody (gemtuzumab) conjugated to ozogamicin, on human MDSCs that improved the efficacy of CAR-T cell immunotherapy [21]. This immunotoxin was investigated in a phase II clinical trial and could significantly deplete CD33+ MDSCs with promising results $[22,23]$. Moreover, anti-GR-1 monoclonal antibody is also able to significantly reduce the MDSC frequency in tumor bearing mice [24, 25]. However it mainly reacted with PMN-MDSCs and thus, MMDSCs could escape from the elimination by Gr-specific antibodies. Recently, Hong Qin et al. generated a novel therapeutic peptibody that efficiently depleted both PMN-MDSCs and M-MDSCs in tumor-bearing mice [26]. Here, a peptibody containing the MDSC-binding peptide and the Fc domain of mouse lgG2a was produced in a eukaryotic system and was characterized in terms of recognition and depletion of MDSCs in $4 \mathrm{~T} 1$ mouse tumor model.

\section{Materials And Methods}

\section{Design and cloning of the peptibody coding sequence}

The MDSC binding peptibody contains a short specific peptide that is linked to the Fc domain of mouse IgG2a antibody (Gene ID: 380793) by a glycine-serine linker (GS linker) together with an IL-2 signal peptide that is located upstream of the sequence (Fig. 1a) [26]. Smal and Bstbl restriction sites were inserted upstream of the peptide and downstream of the linker sequences, respectively. Two slow codon pairs in GS linker were exchanged with a fast counterpart by only a single nucleotide change of $A$ to $T$ (GGAGGC to GGTGGC). In order to produce codon optimized MDSC-specific peptide and linker sequences (84 bp), three overlapping primers were designed and applied in Splicing by Overlap Extension (SOE) PCR (Bioneer, Daejeon, Korea) (Table 1). The product of each PCR was used as a template for the next PCR. The final PCR product was digested with Smal and Bstbl enzymes (Fermentas, Tokyo, Japan) and subsequently cloned into a home-made expression plasmid containing mouse IgG2a Fc and glutamine synthetase (GS) gene as a selection marker. The recombinant construct was ligated using T4 DNA ligase (Fermentas) and transformed into chemically competent $E$. coli DH5a host cells through heat shock approach. Positively transformed colonies were screened by colony PCR using primers designed for CMV promoter (forward primer: 5-CAGACATAATAGCTGACAGACTAAC-3) and SV40 poly-A tail (reverse primer: 5-

ATACCTACCAGTTCTGCGC-3) (Bioneer). The PCR reaction was carried out with an initial melting step at $94^{\circ} \mathrm{C}$ for $5 \mathrm{~min}$, followed by 35 cycles of denaturation at $94^{\circ} \mathrm{C}$ for $30 \mathrm{Sec}$, annealing at $60^{\circ} \mathrm{C}$ for $30 \mathrm{Sec}$, and extension at $72^{\circ} \mathrm{C}$ for $1 \mathrm{~min}$ with a final extension step at $72^{\circ} \mathrm{C}$ for $6 \mathrm{~min}$. Moreover, the selected colonies were confirmed using the enzymatic digestion and DNA sequencing (Applied Biosystems 3500, CA, USA).

Table 1. Overlapping primers applied in SOE PCR to extend MDSC-specific peptide and linker sequences. To produce MDSC specific peptide and linker GS, the two overlapping sequences were designed and fused using the complementary part of sequences (underlined in table) in a PCR cycle. Then, the Smal and Bstbl restriction sites were joined in $5 \nabla$ and $3 \rrbracket$ ends of the product, respectively by overhanging primer pairs 1 and 
2 carrying special annealing sequence of the template molecule ends. Smal restriction site: CCCGGG, Bstbl restriction site: TTCGAA.

\begin{tabular}{|c|c|c|}
\hline $\begin{array}{l}\text { Primer } \\
\text { Name }\end{array}$ & sequence $(5 \rrbracket \rightarrow 3 \rrbracket)$ & $\begin{array}{l}\text { Size } \\
\text { (bp) }\end{array}$ \\
\hline $\begin{array}{l}\text { Overlapping } \\
\text { primer } 1\end{array}$ & TATGGGGCTGGTCTCTGAGCCACGGCTACCAGGTGAAGTCTGGTGGCGGTGG & 52 \\
\hline $\begin{array}{l}\text { Overlapping } \\
\text { primer } 2\end{array}$ & TGATCCGCCGCCACCCGACCCACCTCCGCCCGAGCCACCGCCACCAGACTTCACCT & 56 \\
\hline $\begin{array}{l}\text { Primer } 1, \\
\text { Forward }\end{array}$ & CGGGTATGGGGCTGGTCTCTGA & 22 \\
\hline $\begin{array}{l}\text { Primer } 1, \\
\text { Reverse }\end{array}$ & GAATGATCCGCCGCCACCC & 19 \\
\hline $\begin{array}{l}\text { Primer 2, } \\
\text { Forward }\end{array}$ & TTTTTTCCCGGGTATGGGGCTGGT & 24 \\
\hline $\begin{array}{l}\text { Primer 2, } \\
\text { Reverse }\end{array}$ & CACCATTTCGAATGATCCGCCGCCA & 25 \\
\hline
\end{tabular}

\section{Transient expression of peptibody in $\mathrm{CHO}-\mathrm{K} 1$ cell line}

After cloning of the peptibody sequence, DNA plasmid was gently extracted from the transformed bacterial cells using low endotoxin midiprep kit (Invitrogen, Vilnius, Lithuania). Moreover, CHO-K1 cells were cultured in RPMI-1640 medium (Gibco, Grand Island, NY, USA) supplemented with 10\% fetal bovine serum (Gibco), $100 \mathrm{U} / \mathrm{mL}$ penicillin (Sigma, Darmstadt, Germany), and $100 \mu \mathrm{g} / \mathrm{mL}$ streptomycin (Sigma). Peptibody construct was utilized to transfect CHO-K1 cells according to the manufacturer's instructions (Invitrogen, Carlsbad, CA, USA). Briefly, the plasmid DNA was mixed with P3000 reagent in serum free OPTI-MEM medium (Gibco) and also the lipofectamine3000 was diluted separately in OPTI-MEM medium. The two solutions were mixed and then added to the seeded cells in 12 well plate. Two days after transfection, the transient expression of recombinant peptibody in cell supernatant was checked by ELISA.

\section{Detection of secreted peptibody in cell culture supernatant by ELISA}

Briefly, the ELISA 96-well plate (Nunc, Glostrup, Denmark) was coated with $2 \mu \mathrm{g} / \mathrm{ml}$ of affinity purified sheep anti-mouse IgG polyclonal antibody (SinaBiotech, Tehran, Iran) for 2 hours at $37^{\circ} \mathrm{C}$. After blocking, $100 \mu \mathrm{l}$ 
transfected cell supernatant were added to the wells and incubated for 1.5 hour at $37^{\circ} \mathrm{C}$. The plate was then incubated with horseradish peroxidase-labeled sheep anti-mouse Ig polyclonal antibody (1:1000 dilution in blocking buffer) at $37^{\circ} \mathrm{C}$ for 1 hour. The absorbance values were measured at $450 \mathrm{~nm}$ by an Anthos 2020 Microplate Reader (Biochrom, Cambridge, UK).

\section{Stable expression of the peptibody by transfected $\mathrm{CHO}-\mathrm{K} 1$ cells}

The culture medium was replaced with glutamine free DMEM medium (Sigma) supplemented with 10\% dialyzed FBS (HyClone ${ }^{\text {TM }}$, GE Healthcare, Marlborough, MA, USA) and L-Methionine sulfoximine (MSX, $25 \mu \mathrm{M})$ (Sigma) 48 hours post-transfection. After two weeks, the production level of the peptibody by each colony in the supernatant was measured by ELISA. Colonies with the highest expression level were selected and subcloned in 96-well plates by limiting dilution method. The final clone was cultured in serum free First CHOice medium (UGA Biopharma, Hennigsdorf, Germany) containing Feed alpha and Feed beta supplements (UGA Biopharma) and maintained for two weeks at $31^{\circ} \mathrm{C}$ in $5 \% \mathrm{CO}_{2}$.

\section{Purification of the peptibody from the culture supernatant by protein $\mathrm{G}$ column}

The collected supernatant of stable $\mathrm{CHO}-\mathrm{K} 1$ clone producing the peptibody was centrifuged $(4000 \times \mathrm{g}$ for 5 min at $4^{\circ} \mathrm{C}$ ) and then the clear supernatant was concentrated using a stirred ultra-membrane cell with a 10kDa cut-off (Amicon 8400, Minden, Germany) under N2 gas pressure. The concentrated sample was then passed through a HiTrap Protein G HP column (GE Healthcare). The peptibody was eluted from the protein $\mathrm{G}$ column by a glycine-HCl buffer $(0.1 \mathrm{M}, \mathrm{pH} 2.7)$. The concentration of the peptibody was determined using BCA protein assay kit (Thermo scientific, Rockford, IL, USA).

\section{Characterization of the peptibody by SDS-PAGE and Western blot}

The purified peptibody was mixed with $6 \mathrm{X}$ sample buffer with and without $\beta$-mercaptoethanol and boiled. The protein bands were visualized by Coomassie brilliant blue staining. Moreover, the extracted peptibody was identified by immunoblotting. After the 10\% SDS-PAGE, the protein contents were transferred onto polyvinylidene difluoride (PVDF) membranes in transfer buffer (25 mM Tris; $192 \mathrm{mM}$ glycine; 20\% v/v methanol, $\mathrm{pH} 8.3$ ) at $100 \mathrm{~V}$ for 1 hour (BioRad, Hercules, CA, USA). The membranes were blocked with $5 \%$ skim milk in PBS with $0.05 \%$ Tween20 (PBS-T) and then were individually incubated with HRP-labeled sheep anti-mouse Ig polyclonal antibody (SinaBiotech, Tehran, Iran) in the blocking buffer at 1:1000 dilution under a shaker platform for 1 hour. After repeated washing with PBS-T, the HRP-antibody bound proteins were visualized using DAB substrate kit (BioGenex, Fremont, CA, USA).

\section{Induction of $4 \mathrm{T1}$ breast cancer model}

Female inbred Balb/c mice (6-8 weeks old) were obtained from Pasteur Institute of Iran (Karaj, Iran) and maintained under pathogen-free condition with a 12/12-hour light/dark cycle at $22 \pm 1^{\circ} \mathrm{C}$ with $50 \%$ humidity according to animal welfare guidelines. To induce murine breast tumor, 4T1 cells were cultured in RMPI- 
1640 medium supplemented with 7\% FBS and were subsequently injected into the mammary fat pad of mice at $5 \times 10^{5}$ cells suspended in $50 \mu \mathrm{lPBS}$. All animal experiments were approved by the Ethical Committee of Shahid Beheshti University of Medical Sciences (IR. SBMU. MSP.REC.1396.874).

\section{Determination of binding specificity of peptibody to MDSCs}

To prepare FITC conjugate, the purified peptibody was dialyzed against bicarbonate buffer $(0.1 \mathrm{M}, \mathrm{pH} 8.3)$ overnight at $4^{\circ} \mathrm{C}$. One milligram FITC was dissolved in $1 \mathrm{ml} \mathrm{DMSO}$ and subsequently $20 \mu \mathrm{FITC}$ was mixed with the peptibody $(1 \mathrm{mg})$ in a final volume of $1 \mathrm{ml}$ at room temperature for one hour. Next, the mixture was dialyzed against PBS overnight at $4^{\circ} \mathrm{C}$ [27]. Splenocytes were isolated from 4T1 tumor bearing mice 4 weeks after tumor induction using cell strainer (BD Falcon, Bedford, MA, USA). The splenocytes were then simultaneously incubated with FITC-conjugated peptibody, APC-labeled anti-CD11b (Biolegend, San Diego, $\mathrm{CA}, \mathrm{USA}$ ) and PE-labeled anti-Gr1antibodies (Biolegend) $(1 \mu \mathrm{g} / \mathrm{ml})$ for 1 hour at $4^{\circ} \mathrm{C}$. Also, an irrelevant isotope matched FITC-conjugated antibody (2F9G5 clone) was used as a control for peptibody to exclude the nonspecific binding of peptibody.

\section{In vivo depletion of MDSCs by the peptibody}

The peptibody was administered intraperitoneally at a dose of $50 \mu \mathrm{g}$ per mouse at 17,18 and 19 days after $4 \mathrm{~T} 1$ tumor cell challenge and a control group of mice received PBS alone. On day 20, mice were sacrificed to remove the spleens and tumor tissues. Primary tumors were cut into the $2-4 \mathrm{~mm}$ pieces and then dounced mechanically by a tissue grinder (Kimble Chase, Rockwood, TN, USA) to dissociate into the single cell suspensions. The isolated spleen were gently pressed through a $70 \mu \mathrm{m}$ cell strainer using $1 \mathrm{ml}$ syringe plungers. Then, isolated splenocytes and tumor cells were co-stained with anti-CD11b/Gr1 and FITC conjugated anti-CD45 antibodies (Biolegend). FACS analysis was performed using flow cytometer cell analyzer (BD Biosciences, FACS Lyrics, San Jose, CA, USA) and the data was analyzed by Flow Jo software V10 (Tree Star Inc, Ashland, OR, USA).

\section{Statistical analysis}

Statistical analyses were conducted by Graph Pad Prism 8 software (Graph Pad Software Inc., San Diego, CA, USA) using two-tailed $t$-test to compare MDSC percentages in treated group of mice with the control group that received PBS. P value $<0.05$ was defined statistically significant and represented as *.

\section{Results}

\section{MDSC binding peptide production in eukaryotic $\mathrm{CHO}$-K1 cells}

The sequence of the MDSC binding peptide and the GS linker were assembled by SOE-PCR which resulted in production of a $111 \mathrm{bp}$ fragment (Fig. 1b). The amplified sequence was then inserted into the plasmid that carried the coding sequences of mouse IgG2a Fc protein. Transformed $E$. coli colonies harboring the correct construct had a 1317 bp amplicon in colony PCR using specific primers (Fig. 1C). Two selected colonies (C12 and C13) were confirmed using enzymatic digestion (Fig. 1d). Furthermore, DNA sequencing 
analysis of these two colonies indicated that the MDSC binding peptide was successfully cloned into the expression vector in a correct frame shift without any mutation.

It is interesting to mention that before codon pair optimization of GS linker sequence, the maximum transient expression level of the transfected cells was $12 \mathrm{ng} / \mathrm{ml}$ despite several attempts to improve the production level. Codon pair optimization of the MDSC-peptide coding and GS linker sequences improved peptibody production level by 20.8 folds $(250 \mathrm{ng} / \mathrm{ml})$ after transient transfection. Finally, after multiple rounds of subcloning and selection of stable clones, a stable high producer clone which was producing 8 $\mu \mathrm{g} / \mathrm{ml}$ of the peptibody was selected and expanded.

\section{Characterization of MDSC binding peptide using SDS-PAGE and immunoblotting}

The size and structure of the purified peptibody were investigated by SDS-PAGE and the peptibody migrated as a single band. The molecular weight of the peptibody was around $70 \mathrm{kDa}$ under non-reducing conditions and its reduced form showed a smaller $35 \mathrm{kDa}$ band (Fig. 2a). The recombinant peptibody was also detected by immunoblotting with HRP conjugated sheep anti-mouse IgG as a 70 kDa protein band (Fig. 2b).

We next determined the binding characteristics of the peptibody to MDSCs. When the 4T1 tumor size reached $760 \mathrm{~mm}^{3}$, the splenocytes were harvested and stained with the FITC-conjugated peptibody and APC-anti-CD11b and PE-anti-Gr1 antibodies as MDSC markers. The peptibody bound to $98.8 \%$ of CD11b/Gr1 positive MDSCs, however, the control peptibody failed to recognize this cell population (Fig. 3). The CD $45 / C D 11 \mathrm{~b} / \mathrm{Gr}-1$ positive MDSCs substituted $66.2 \%$ of the splenocytes obtained from the $4 \mathrm{~T} 1$ tumor bearing mice.

\section{MDSCs depletion by the peptibody in 4T1 tumor model}

The peptibody was intraperitoneally administrated at $50 \mu \mathrm{g} / \mathrm{mouse} /$ day; 17,18 and 19 days after induction of $4 \mathrm{~T} 1$ tumors (tumor volume: $1500 \mathrm{~mm}^{3}$ ). Flow cytometric analysis showed that intratumoral MDSCs were significantly depleted after peptibody treatment compared with the control group that received PBS alone (39.6\% compared to 57.3\%) ( $\mathrm{P}<0.05)$ (Fig. 4). However, no significant reduction of MDSCs was observed in splenocytes of tumor bearing mice (Fig. 4).

\section{Discussion}

It is well known that tumor microenvironment is infiltrated with various types of immune suppressive cells including the immature myeloid cells, MDSCs [28]. MDSCs certainly have critical roles in tumor development and are documented as a major immunosuppressive population against efficient immunotherapy $[29,30]$. In this context, MDSC-targeting strategies lead to MDSC depletion or inhibition of their immunosuppressive function in malignancies. ATRA, an isomer of retinoic acid, can mediate differentiation of MDSCs to mature cells such as macrophages, dendritic cells and granulocytes in human and mouse tumors. By induction of MDSC differentiation, ATRA enhances anti-tumor immune responses of $\mathrm{CD} 4^{+}$and $\mathrm{CD} 8^{+} \mathrm{T}$ cells and improves the efficacy of cancer vaccines [31]. In a lymphoma model, 5- 
fluorouracil and gemcitabine dramatically decreased MDSC numbers in spleen and tumor tissues. This inhibition of MDSC population was associated with induction of the INFY production in tumor-specific CD $8^{+}$ T cells and development of anti-tumor T cell dependent immune responses [32, 33]. In addition, anti-Gr-1 monoclonal antibody eliminates $\mathrm{Gr}-1^{+}$granulocytic MDSCs and can restore the antitumor $\mathrm{T}$ cell responses. However, it causes elimination of mature granulocytes and consequently induces a severe systemic immunosuppression [34]. Thus, the finding of a specific MDSC-depleting agent with poor off-targeting activity is valuable to overcome the robust tumor-induced immunosuppression and leading to enhancement of cancer immunotherapies in clinical practice. In order to discover an efficient MDSC-targeting agent, Hong Qin et al. identified peptibodies by phage display technique that efficiently depleted both PMN-MDSCs and M-MDSCs in lymphoid peripheral organs and intratumoral sites [26].

In this study, we produced an anti-MDSC peptibody containing a MDSC-binding peptide fused to Fc domain of mouse IgG2a by a GS linker in CHO-K1 cells [26]. Purified peptibody showed a $70 \mathrm{kDa}$ dimeric protein in immunoblotting that was dissociated into $35 \mathrm{kDa}$ monomeric subunits under reducing condition. In our first attempt, the MDSC-specific peptide and linker sequences were synthetized (Biomatik) and cloned in a home-made vector harboring FC domain of mouse IgG2a. However, we found that even optimization was not efficient enough for expression in both transient and stable transfected $\mathrm{CHO}-\mathrm{K} 1$ cells. Then, the sequences of MDSC-binding peptide and GS linker were optimized on the basis of codon pair bias theory to improve the expression level of peptibody [35] (Supplementary Table 1). We exchanged the slow codon pairs with a fast counterpart in GS linker by only a single nucleotide change of A to T (GGAGGC to GGTGGC). In agreement with Trinh study [35], we observed that a fast codon pair improved peptibody production levels from $12 \mathrm{ng} / \mathrm{ml}$ to $250 \mathrm{ng} / \mathrm{ml}$ in the CHO-K1 transiently transfected host. Our efforts to produce stable cell line with original sequences also led to increased production to $8 \mathrm{ug} / \mathrm{ml}$. Thus, in both transient and stable transfection plans, codon pair optimization resulted in a significant improvement in peptibody production level.

It was suggested that overrepresented codon pairs may reduce efficiency of transition process and may thus act as ribosomal pause sites. These slow pairs give the intact polypeptides an opportunity to fold properly by chaperones. So, it seems that elimination of the slow codon pairs to accelerate the translation step may result in increasing the yield of recombinant protein production [36, 37].

When 4T1 murine mammary carcinoma cells were implanted orthotopically into mammary fat pad of female Balb/c mice, they could spontaneously metastasize to bone, liver, lungs and spleen organs which was also affected in late-stage cancer patients $[38,39]$. The invasive 4T1 tumor growth causes a leukemoid reaction and splenomegaly accompanied by massive myeloid cell infiltration that was partly mediated via colony-stimulating factor and chemokines secreted by $4 \mathrm{~T} 1$ tumor cells [40]. The predominant population of these leukocytes has been reported to be $\mathrm{CD} 11 \mathrm{~b}^{+} / \mathrm{Gr}-1^{+}$immature myeloid MDSCs that accumulate in primary tumors, spleen, lung and peripheral blood $[30,41]$. Transcripts of G-CSF and GM-CSF and myeloid cell chemokines MCP-1, KC, RANTES, MIP-1a and MIP-1b have been detected in cultured 4T1 cells in vitro which suggests that $4 \mathrm{~T} 1$ tumor cells individually cause a remarkable increase in myeloid infiltrations of primary tumors and metastatic foci. Our findings consistently showed that 4T1 tumor promoted the MDSCs 
expansion in spleen and tumor beds [40]. Interestingly, in our experiments 4T1 induced MDSCs to constitute $89 \%$ and $57 \%$ of $C D 45^{+}$population in spleen and primary tumor, respectively, four weeks after tumor inoculation (Fig. 4). This suppressive population probably participates in establishment and survival of 4T1 tumors within 3-4 weeks using blocking of innate and adaptive anti-tumor immune responses directed against $4 \mathrm{~T} 1$ cell antigens and as a result reduction of immune surveillance.

The purified peptibody bound to $98.8 \%$ of splenic MDSCs isolated from 4T1 tumor implanted mice and could deplete MDSC population in vivo. Intraperitoneal injection of three doses of peptibody significantly reduced intratumoral MDSC numbers but this reduction was not significant for splenic MDSCs. One explanation for this finding is that four weeks after tumor inoculation the MDSCs constitute $89 \%$ of the leukocytes in spleen. Thus, administration of 3 doses of peptibody may not be sufficient to induce significant reduction of MDSCs. However, the frequency of MDSCs in tumor microenvironment is not so high to counteract the peptibody depletion effects. The dose and stability of the peptibody administered into the peritoneum might also affect its functional failure in the spleen. Assessment of the effect of peptibody on MDSCs at early stages of tumor development, during the first 2 weeks of implantation, may help to elucidate the underlying reasons.

\section{Conclusion}

In this study, we successfully constructed and produced a fusion protein containing MDSC-binding peptide in $\mathrm{CHO}-\mathrm{K} 1$ cells and improved the expression level of the peptibody by codon pair optimization in GS linker. The peptibody was able to deplete MDSC population resident in 4T1 tumor microenvironment. This peptibody could be applied for combination immunotherapy to overcome the MDSC immune suppressive function.

\section{Declarations}

Ethics approval and consent to participate: Not applicable

Consent for publication: Not applicable

\section{Availability of data and materials:}

All data generated or analyzed during this study are included in the manuscript.

\section{Competing interests:}

The authors declare that the research was conducted in the absence of any commercial or financial relationships that could be construed as a potential conflict of interest.

\section{Funding:}

This work was financially supported by Iran National Science Foundation (INSF) (grant number: 96006157), Shahid Beheshti University of Medical Sciences (grant number: 12442), Mazandaran University of Medical 
Sciences (grant number: 1376), and Avicenna Research Institute (grant number: 96-017).

\section{Authors' contributions:}

KRA: performing the tests, data collection, analysis, and writing original draft, VKB: participating in experiments, and data analysis, JM: participating in experiments, and advising, HAO: investigation, methodology, project administration, supervision, FS: methodology, project administration, and supervision, MHF: methodology, data analysis, MJT: data curation, formal analysis, investigation, methodology, project administration, supervision, validation, and editing, MS: conceptualization, data curation, formal analysis, investigation, methodology, project administration, supervision, validation, writing and editing. All authors approved the final manuscript submitted to the journal.

\section{Acknowledgements}

This work was financially supported by Iran National Science Foundation (INSF) (grant number: 96006157), Shahid Beheshti University of Medical Sciences (grant number: 12442), Mazandaran University of Medical Sciences (grant number: 1376), and Avicenna Research Institute (grant number: 96-017). This work is part of the PhD thesis of Khadijeh Ramezani- Ali Akbari.

\section{Disclosure of potential conflicts of interest: Not applicable}

\section{Research involving Human Participants and/or Animals:}

All animal experiments were approved by the Ethical Committee of Shahid Beheshti University of Medical Sciences (IR. SBMU. MSP.REC.1396.874). All applicable international, national, and/or institutional guidelines for the care and use of animals were followed. All procedures performed in this study were in accordance with the ethical standards of the institutional and/or national research committee and with the 1964 Helsinki declaration and its later amendments or comparable ethical standards.

Informed consent: Not applicable

\section{References}

1. Gabrilovich D, Nagaraj S (2009) Myeloid-derived suppressor cells as regulators of the immune system. Nat Rev Immunol 9:74-162

2. Gallina G, Dolcetti L, Serafini $P$ et al (2006) Tumors induce a subset of inflammatory monocytes with immunosuppressive activity on CD8+ T cells. J Clin Invest 116(10):2777-2790

3. Serafini P, Borrello I, Bronte V (2006) Myeloid suppressor cells in cancer: recruitment, phenotype, properties, and mechanisms of immune suppression. Semin Cancer Biol 16(1):53-65

4. Bronte V, Brandau S, Chen S-H et al (2016) Recommendations for myeloid derived suppressor cell nomenclature and characterization standards. Nat Commun 7(1):1-10

5. Almand B, Clark Jl, Nikitina E et al (2001) Increased Production of Immature Myeloid Cells in Cancer Patients: A Mechanism of Immunosuppression in Cancer. J Immunol 166(1):678-689 
6. Gabrilovich DI, Bronte V, Chen S-H et al (2007) The terminology issue for myeloid-derived suppressor cells. Cancer Res 67(1):425

7. Gabrilovich DI (2017) Myeloid- derived suppressor cells. Cancer Immunol 5(1):3-8

8. Wang Y, Ding Y, Guo N et al (2019) MDSCs: Key criminals of tumor pre-metastatic niche formation. Front Immunol 10(172):1-16

9. Kumar V, Patel S, Tcyganov E et al (2016) The nature of myeloid-derived suppressor cells in the tumor microenvironment. Trends Immunol 37(3):208-220

10. Meyera C, Sevko A, Ramacher M et al (2011) Chronic inflammation promotes myeloid-derived suppressor cell activation blocking antitumor immunity in transgenic mouse melanoma model. PNAS 108(41):17111-6

11. Lu Z, Zou J, Li S et al (2020) Epigenetic therapy inhibits metastases by disrupting premetastatic niches. Nature 579(7798):284-290

12. Orecchioni S, Talarico G, Labanca V et al (2018) Vinorelbine, cyclophosphamide and 5-FU effects on the circulating and intratumoural landscape of immune cells improve anti-PD-L1 efficacy in preclinical models of breast cancer and lymphoma. Br J Cancer 118(10):1329-1336

13. Kim NR, Kim YJ (2019) Oxaliplatin regulates myeloid-derived suppressor cell-mediated immunosuppression via downregulation of nuclear factor-KB signaling. Cancer Med 8(1):276-288

14. Fleming V, Hu X, Weber $\mathrm{R}$ et al (2018) Targeting myeloid-derived suppressor cells to bypass tumorinduced immunosuppression. Front Immunol 9(398):1-11

15. Bauer R, Udonta F, Wroblewski M et al (2018) Blockade of myeloid-derived suppressor cell expansion with all-trans retinoic acid increases the efficacy of antiangiogenic therapy. Cancer Res 78(12):32203232

16. Nefedova Y, Fishman M, Sherman S et al (2007) Mechanism of all-trans retinoic acid effect on tumorassociated myeloid-derived suppressor cells. Cancer Res 67(22):11021-8

17. Alizadeh D, Trad M, Hanke NT et al (2014) Doxorubicin eliminates myeloid-derived suppressor cells and enhances the efficacy of adoptive T-cell transfer in breast cancer. Cancer Res 74(1):104-118

18. Marigo I, Dolcetti L, Serafini P et al (2008) Tumor-induced tolerance and immune suppression by myeloid derived suppressor cells. Immunol Rev 222(1):162-179

19. Suzuki E, Kapoor V, Jassar AS et al (2005) Gemcitabine selectively eliminates splenic Gr-1+/CD11b + myeloid suppressor cells in tumor-bearing animals and enhances antitumor immune activity. Clin Cancer Res 11(18):6713-6721

20. Ko H-J, Kim Y-J, Kim Y-S et al (2007) A combination of chemoimmunotherapies can efficiently break self-tolerance and induce antitumor immunity in a tolerogenic murine tumor model. Cancer Res 67(74):77-86

21. Fultang L, Panetti S, Ng M et al (2019) MDSC targeting with Gemtuzumab ozogamicin restores T cell immunity and immunotherapy against cancers. EBioMedicine 47:235-246

22. Fournier E, Duployez N, Ducourneau B et al (2020) Mutational profile and benefit of gemtuzumab ozogamicin in acute myeloid leukemia. Blood 135(8):542-6 
23. D.Bernstein RAppelbaumF I (2017) Gemtuzumab ozogamicin for acute myeloid leukemia. Blood 130(22):2373-6

24. K.Srivastava M, Zhu L, Harris-White M et al (2012) Myeloid suppressor cell depletion augments antitumor activity in lung cancer. PLoS ONE 7(7):40677-90

25. Hurez V, Daniel BJ, Sun L et al (2012) Mitigating age-related immune dysfunction heightens the efficacy of tumor immunotherapy in aged mice. Cancer Res 72(8):2089-2099

26. Qin H, Lerman B, Sakamaki I et al (2014) Generation of a new therapeutic peptide that depletes myeloid-derived suppressor cells in tumor-bearing mice. Nat Med 20(6):676-681

27. Holmes KL, Lantz LM (2001) Protein labeling with fluorescent probes. Methods Cell Biol 63:185-204

28. Bronte V, Serafini P, Apolloni E et al (2001) Tumor-Induced Immune Dysfunctions Caused by Myeloid Suppressor Cells. J Immunother 24(6):431-446

29. Younos I, Donkor M, Hoke T et al (2011) Tumor- and organ-dependent infiltration by myeloid-derived suppressor cells. Int Immunopharmacol 11(7):816-826

30. Youn J-I, Nagaraj S, Collazo M et al (2008) Subsets of Myeloid-Derived Suppressor Cells in TumorBearing Mice. J Immunol 181(8):5791-5802

31. Kusmartsev S, Cheng F, Yu B et al (2003) All-trans-retinoic acid eliminates immature myeloid cells from tumor-bearing mice and improves the effect of vaccination. Cancer Res 63(15):4441-9

32. Vincent J, Mignot G, Chalmin F et al (2010) 5-Fluorouracil selectively kills tumor-associated myeloidderived suppressor cells resulting in enhanced $\mathrm{T}$ cell-dependent antitumor immunity. Cancer Res 70(8):3052-3061

33. Le HK, Graham L, Esther $C$ et al (2009) Gemcitabine directly inhibits myeloid derived suppressor cells in BALB/c mice bearing 4T1 mammary carcinoma and augments expansion of T cells from tumorbearing mic. Int Immunopharmacol 9(78):900-909

34. ronte B, Wang V, Overwijk $\mathrm{M}$ et al (1998) Apoptotic death of CD8+ T lymphocytes after immunization: induction of a suppressive population of Mac-1+/Gr-1+ cells. J Immunol 161(10):5313-5320

35. Trinh R, Gurbaxani B, Morrison SL et al (2004) Optimization of codon pair use within the (GGGGS) "3 linker sequence results in enhanced protein expression. Mol Immunol 40(10):717-722

36. Quax EF, Claassens TJ, Söll N et al (2015) Codon Bias as a Means to Fine-Tune Gene Expression. Mol Cell 59(2):149-161

37. Sharp PM, Li WH (1986) An evolutionary perspective on synonymous codon usage in unicellular organisms. J Mol Evol 24(12):28-38

38. Yoneda T, Michigami T, Yi B et al (2000) Actions of bisphosphonate on bone metastasis in animal models of breast carcinoma. Cancer 88(12):2979-2988

39. Lelekakis M, Moseley JM, Martin TJ et al (1999) A novel orthotopic model of breast cancer metastasis to bone. Clin Exp Metastasis 17:168-170

40. DuPre' S A, W.Hunter K (2007) Murine mammary carcinoma 4T1 induces a leukemoid reaction with splenomegaly: Association with tumor-derived growth factors. Exp Mol Pathol 82(1):12-24 
41. Ostrand-Rosenberg S (2010) Myeloid-derived suppressor cells: more mechanisms for inhibiting antitumor immunity. Cancer Immunol Immunother 59:1593-1600

\section{Figures}
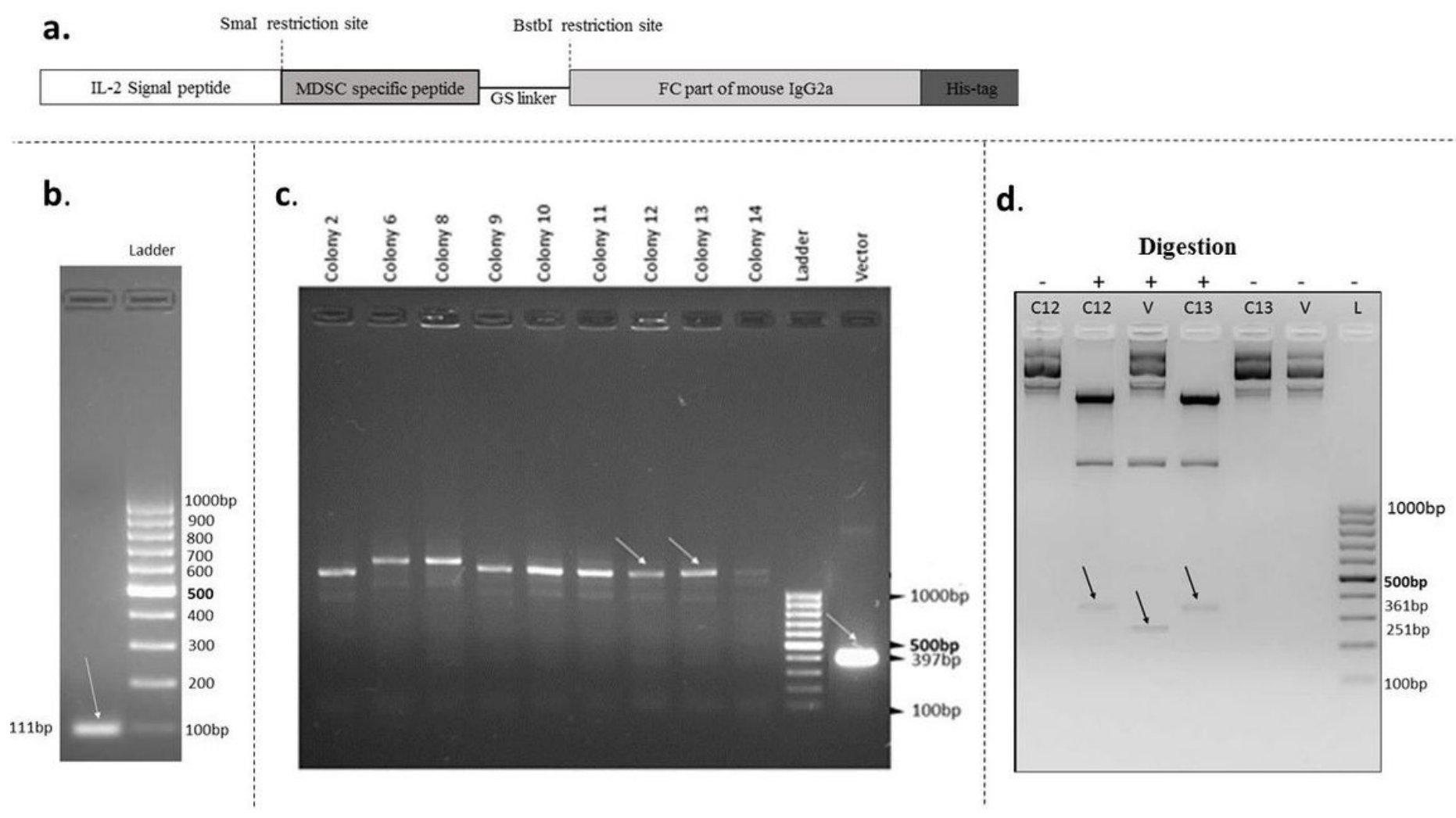

Figure 1

Design, cloning and confirmatory data on MDSC specific peptibody construct. a) Peptibody contained MDSC-binding peptide fused to FC domain of mouse IgG2a by a glycine-serine linker (GS linker). His-tag and IL-2 signal peptide coding sequences were added to $3^{\prime}$ and $5^{\prime}$ ends of peptibody, respectively. b) Electrophoresis of SOE-PCR product contained coding sequence of a MDSC-binding peptide and a GS linker. Target amplicon had a 111 bp size after electrophoresis on $2 \%$ agarose gel (marked by white arrow). c) Colony PCR of transformed colonies with peptibody construct. After electrophoresis, the positive colonies had a $1317 \mathrm{bp}$ fragment on 1\% agarose gel in comparison to the mock vector (397 bp fragment) (pointed by white arrows). d) Double digestion of DNA plasmids extracted from two selected colonies using HindIII and Sall. The digested constructs having the MDSC binding peptide were cleaved to a $361 \mathrm{bp}$ fragment but digested mock vector had a smaller band (251 bp fragment) on $2 \%$ agarose gel (pointed by dark arrows). C12: colony 12; C13: colony 13; V: mock vector contained FC-domain, and L: 100 bp ladder (Invitrogen) 
a.

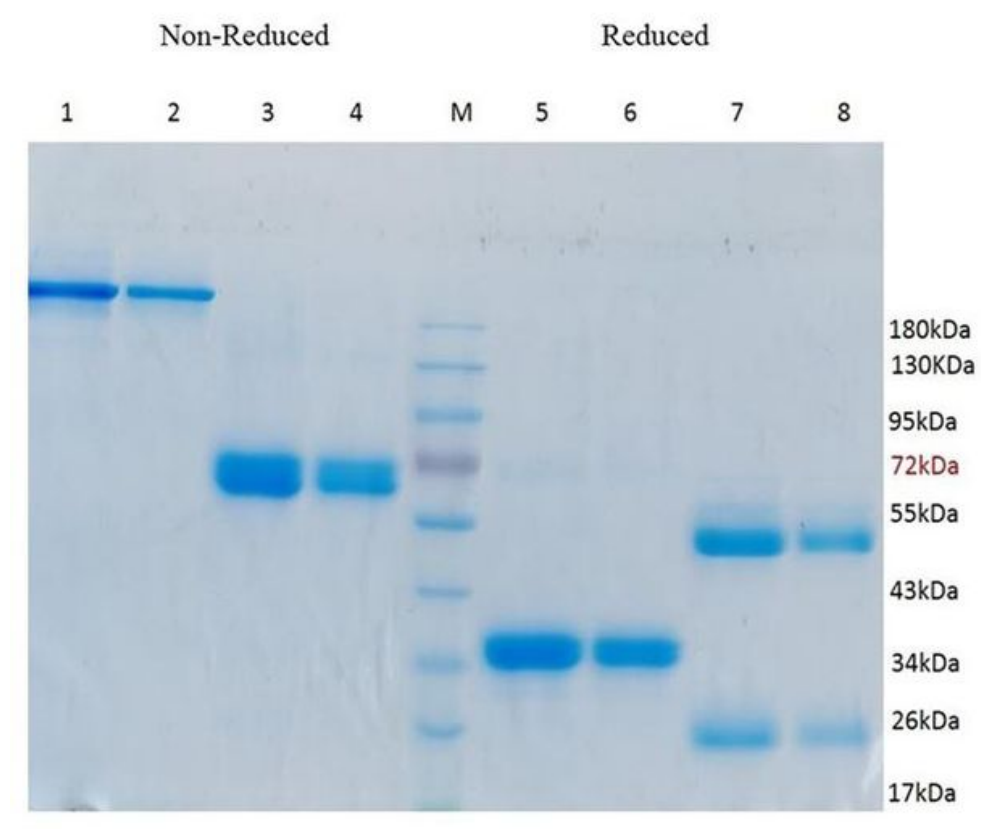

b.

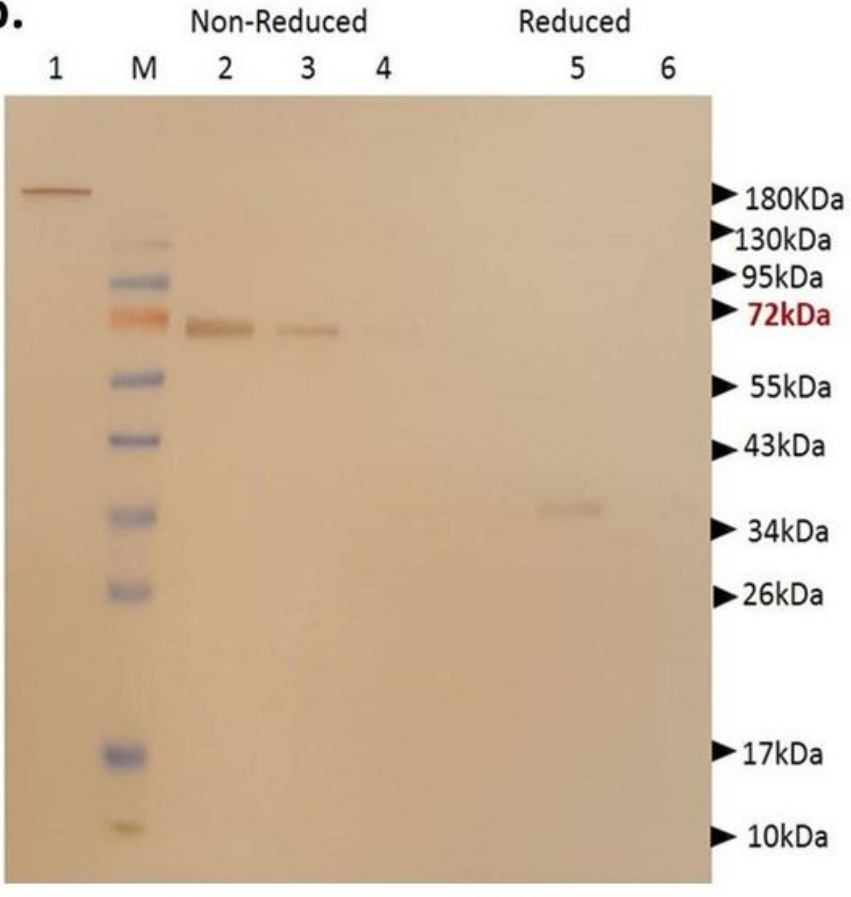

\section{Figure 2}

Characterization of the purified peptibody by SDS-PAGE analysis and Immunoblotting. a) Peptibody was purified using protein $\mathrm{G}$ column and then separated on 10\% SDS-PAGE gel in reducing and non-reducing conditions. Non-Reduced: lane 1: mouse IgG $(10 \mu \mathrm{g})$; lane 2: mouse lgG $(5 \mu \mathrm{g})$; lane 3: purified peptibody $(10 \mu \mathrm{g})$; lane 4: purified peptibody $(5 \mu \mathrm{g}) ;$ M: PageRuler Prestained Protein Ladder (Fermentas). Reduced: lane 5: peptibody $(10 \mu \mathrm{g})$; lane 6: peptibody $(5 \mu \mathrm{g})$; lane 7: mouse $\operatorname{lgG}(10 \mu \mathrm{g})$; lane 8: mouse $\lg \mathrm{g}(5 \mu \mathrm{g})$. b) Immunoblotting of purified peptibody by HRP-labeled sheep anti-mouse IgG. Transferred peptibody was visualized by DAB substrate kit under reducing and non-reducing conditions. Non-reduced: lane 1: mouse IgG $(0.2 \mu \mathrm{g} /$ well); lane 2: peptibody $(0.4 \mu \mathrm{g} /$ well); lane 3: peptibody $(0.2 \mu \mathrm{g} /$ well $)$; lane 4: peptibody $(0.1$ $\mu \mathrm{g} /$ well); M: PageRuler Prestained Protein Ladder (Fermentas). Reduced: lane 5: peptibody ( $0.4 \mu \mathrm{g} / \mathrm{well})$; lane 6: peptibody $(0.2 \mu \mathrm{g} /$ well $)$ 

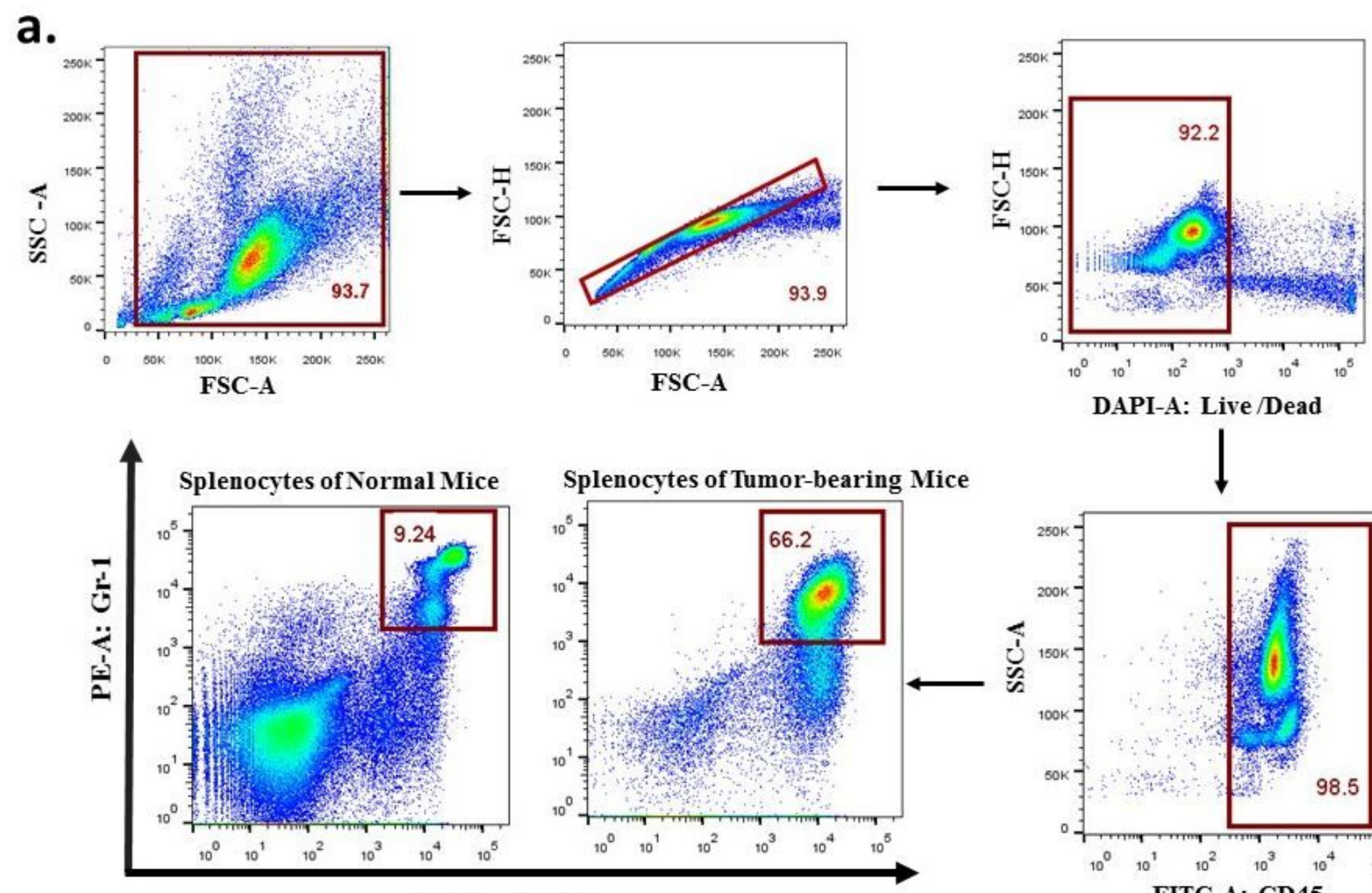

APC-A: CD11b
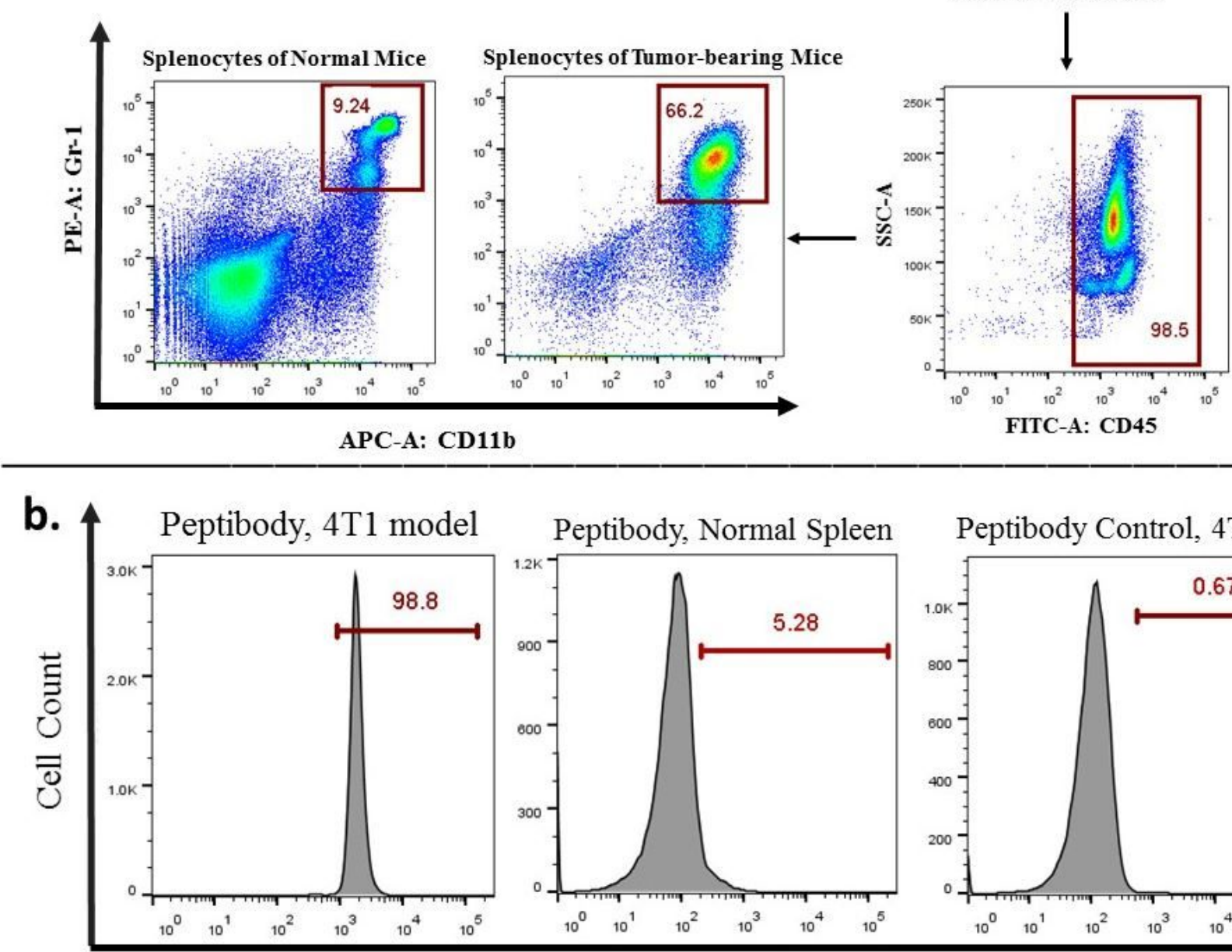

Peptibody Control, 4T1 model

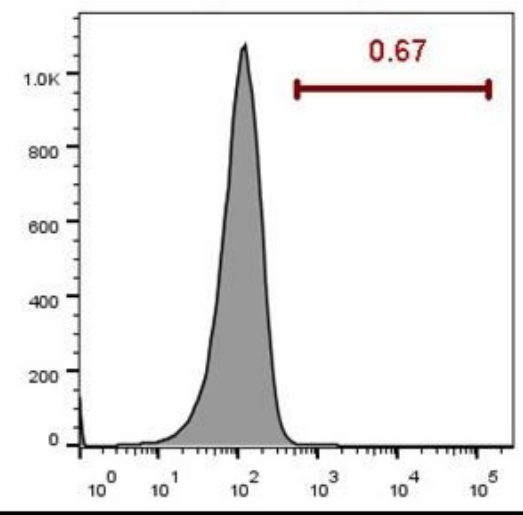

Fluorescence Intensity

Figure 3

Flow cytometric analysis of capability of peptibody binding to splenic MDSCs. a) Splenic MDSCs were identified as $\mathrm{CD}_{4} 5^{+} / \mathrm{CD} 11 \mathrm{~b}^{+} / \mathrm{Gr}-1^{+}$population and accumulated in $4 \mathrm{~T} 1$ bearing mice $(66.2 \%)$ in comparison to normal mice (9.24\%) (3 mouse pre group). b) To evaluate binding ability of peptibody to MDSCs, FITCconjugated peptibody, APC-anti-CD11b and PE-anti-Gr-1 antibodies were used to stain the splenocytes isolated from 4T1 bearing and normal mice. Also, a FITC-conjugated control antibody was applied to exclude nonspecific bindings. FITC intensity was determined in the gated MDSCs and represented as the histograms. Peptibody bound to $98.8 \%$ of splenic MDSCs in 4 T1 mice but recognized only $5.28 \%$ of splenocytes in normal mice 
a.

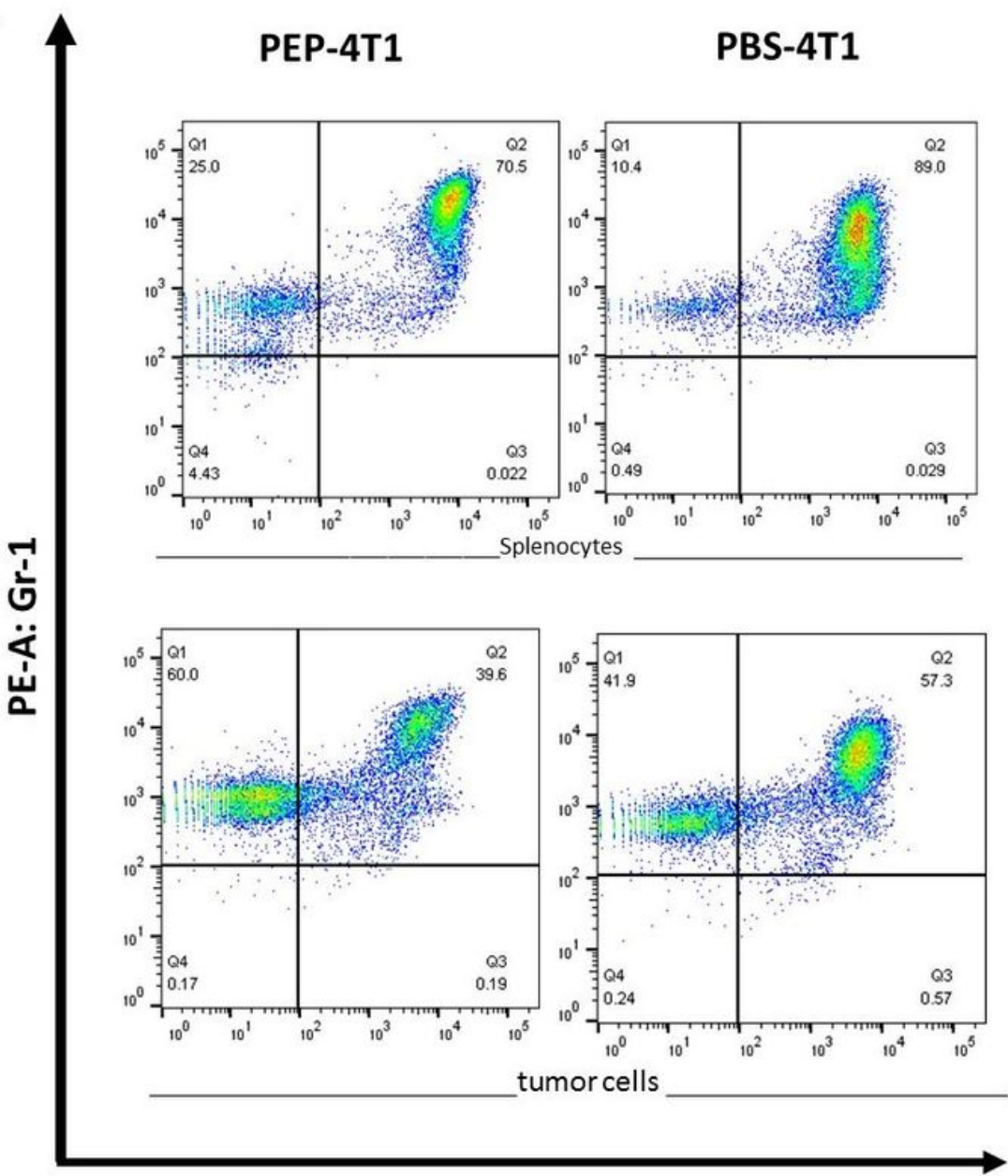

APC-A:CD11b b.

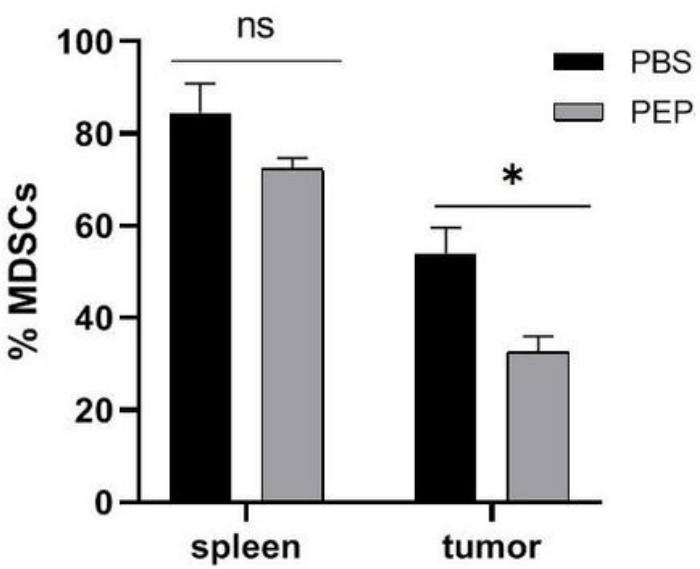

Figure 4

Flow cytometric analysis of MDSCs in the spleen and primary tumor of 4T1 bearing mice after treatment with peptibody. Peptibody was intraperitoneally injected at $50 \mu \mathrm{g}$ dose per day for three days, and then spleens and tumors were harvested to analyze by flow cytometry. Control group was given PBS and in each group was included 4 female Balb/c mouse. Peptibody treatment could decrease the splenic MDSCs to $70.5 \%$ proportion compared to PBS received group and significantly depleted the intratumoral MDSCs to $39.6 \%(a, b)$. PEP: peptibody

\section{Supplementary Files}

This is a list of supplementary files associated with this preprint. Click to download.

- SupplementaryTable.1.docx 